\title{
A REVIEW- SUPER CAPACITOR SYSTEMS AND ITS PERFORMANCE
}

\author{
Raji George $^{1}$, Ajay Rijal ${ }^{2}$, Bhargava ${ }^{3}$, Raj Jung Mahat ${ }^{4}$, Santosh Yadav $^{5}$ \\ ${ }^{1}$ Professor, Department of Mechanical Engineering, MSRIT, Bangalore \\ ${ }^{2,3,4,5}$ Final Year UG Student, Department of Mechanical Engineering, MSRIT
}

\begin{abstract}
Researchers worldwide are in quest for developing newer energy storing devices as well as looking for improving existing devices in their performance. Demerit of capacitor is the low energy density and that for a battery is the low power density, these two difficulties are overcome by super capacitor which is the latest addition to energy storing devices. The present paper highlights on the differences between capacitor, battery and super capacitor, bringing out the ability of ultra-capacitor. The components of super-capacitor system are described in detail and focusing on the pros and cons of different material used for these components and their adoptability. The performance of the system is also dealt in detail with respect to energy density, power density, specific capacitance, self-discharge and cycle life.
\end{abstract}

Keywords: Super Capacitor, Energy Density, Power Density, Specific Capacitance, Cycle Life.

\section{INTRODUCTION}

Super capacitors also known as ultra-capacitors are the latest addition to the energy storage devices. These systems have enormous power, adequate energy density with longer cycle life. It lies between normal capacitor and a battery. These systems have found tremendous application in the field of electronics, hybrid electric vehicles and domestic electronics consumer items.

Although extensive research is been carried out in the field of super-capacitors still there are many unanswered questions and scope for optimization. Super-capacitors has numerous components which individually and synergistically contributes to its performance ergo optimization of each its component is essential. However individual component optimization will not ensure the optimization of system as a whole hence an attempt is made to study individual components and their synergic effect when put together. Experimental data and observations made by researchers are correlated to the underlying principles of energy sciences and proper conclusions and reasoning is derived for each mechanism and data presented.

Major parameters that describe the performance of an energy storing device are power density, energy density, Self discharge and cycle life. Each of this parameter is different for capacitor, super-capacitor and batteries.

Power density is defined as the amount of energy per second that could be supplied by 1 gram of active material at the time of discharge and any energy storing device is expected to have high power density. Power density for Capacitor is $\left(10^{4}\right.$ to $\left.10^{6} \mathrm{KW} / \mathrm{kg}\right)$, which is highest among the energy storage device, and is least for batteries (lithium ion battery having power density in range of 0.4 to $3 \mathrm{KW} / \mathrm{kg}$ ) [1]. Super capacitor has intermediate power density, for symmetric super-capacitor $(5 \mathrm{KW} / \mathrm{kg} \quad-55 \quad \mathrm{KW} / \mathrm{kg})$ [2] and for asymmetric super-capacitor (1 to $5 \mathrm{KW} / \mathrm{kg}$ ) [2].
Energy density for super capacitor is defined as the amount of energy stored in 1 gram of the active electrode material. In the case of energy density Batteries have highest energy density (for lithium ion (120 to 200) $\mathrm{Wh} / \mathrm{kg}$ ) followed by super capacitor with energy density $4-8 \mathrm{Wh} / \mathrm{kg}$ for symmetric super-capacitor and 10 to $50 \mathrm{Wh} / \mathrm{kg}$ for asymmetric super-capacitor. Capacitor has very low energy density value of $(0.01$ to $0.05 \mathrm{Wh} / \mathrm{kg})$ [2]. In batteries energy density is directly proportional to voltage and voltage is constant throughout its charging and discharging process. Electrode potential only depends on composition of electrode material and electrolyte [3]. In the case of supercapacitor voltage depends on state of charge and on charging the potential of electrode starts gradually increasing and reaches that of source at the end of charge cycle [3].

Cycle life is defined as number of charge -discharge cycles for which super-capacitor is able to perform without any observable degradation. Capacitor has highest cycle life followed by super capacitor with cycle life of $100000-$ 500000 cycles [2]. Batteries have low cycle life (1000 cycles), owing to the faradic reaction that happens in the electrode, as compared to capacitors and super-capacitors.

In Batteries energy is stored in electrode through redox reaction also know $\mathrm{n}$ as faradic reaction which involves loss and gain of electron to form new chemical product on electrode such that electrode composition while charging and discharging is different [4]. As energy storage involves faradic redox reaction they involve thermodynamic limitation and consist of some kind of irreversibility [5]. Owing to the redox reaction it takes long time to charge [5].

Electrochemical Capacitor and EDLC (Electric Double Layer Capacitor) super-capacitor works on same principle of electrostatic attraction, however due to the large active surface area of EDLC super-capacitor (4 to $50 \mathrm{Wh} / \mathrm{kg}$ ) the 
amount of energy that it can store is large in comparison to electrostatic capacitor $(0.01$ to $0.05 \mathrm{Wh} / \mathrm{kg}$ ) [6].

Super capacitor are generally of two types EDLC and pseudo type, their concepts are intriguing and researcher are trying to improve their performance in terms of energy density and power density so that it can be used in existing electronic products [3]

EDLC works on the basic principle of electrostatic capacitor that is electrostatic attraction of charges. When potential is applied across the electrode there is movement of solvated ions, i.e. negative ions move towards the positive electrode and vice versa and form Helmholtz double layer which is also known as diffusive layer. This configuration as a whole resembles two capacitors $\left(C_{1}\right)$ and $\left(C_{2}\right)$ connected in series.

The equivalent capacitance (C) is given by

$\frac{1}{c}=\frac{1}{c_{1}}+\frac{1}{c_{1}}$

For an EDLC super-capacitor Capacitance (c) depends on electrode surface area (A) and distance between the positive and negative electrode (d) [7].

$C=\varepsilon \frac{a}{d}$

Energy density for EDLC super-capacitor is same as that for capacitor, it depends on capacitance (c) and square of voltage (v) and is given by [7]

$E=\frac{1}{2} c v^{2}$

From the above equation the amount of energy that can be stored per gram of electrode is directly proportion to square of operating voltage and specific capacitance but operating voltage is limited by type of electrolyte. Voltage window for aqueous electrolyte is $(1.2$ to $1.63 \mathrm{~V})$ and for organic electrolyte is (2 to $2.8 \mathrm{~V}$ ) [1].

Power density of super-capacitor depends on equivalent series resistance (ESR) which in turn depends on the electrode resistance, type of electrolyte and resistance of separator. [7]

$P=\frac{\mathrm{V}^{2}}{4 R_{28 F^{2}}}$

Pseudo capacitor works in the principle of fast faradic reaction between the electrolyte and electro active species of electrode (functional groups and many more). In normal EDLC super-capacitors also in a small extent pseudo capacitance might be present due to impurities in electrode and electrolyte. The pseudo capacitance(c) is measured in terms of amount of charge stored for an applied potential (V). [4]

$C=\frac{d g}{d v}$
Like batteries they also possess thermodynamic irreversibility. Pseudo capacitance can occur due to redox reaction (chemically reacts to form new products), Electro absorption (under potential deposition) and intercalation (insertion of ion in the gap [6].

Pseudo capacitance is mainly due to intercalation and also due to functional groups present in electrode material which takes part in the redox reaction [8]. Presence of water on electrolyte in trace amount also helps in redox reaction. It is found that water in electrolyte encourages the formation of decomposition product via side reaction which will affect the performance of super-capacitor [6].

\section{SUPER CAPACITOR COMPONENTS}

Major component of super capacitor storage device are electrode, electrolyte, separator and collector. The understanding of the behavior and properties of these components are the most critical and will help in developing an efficient super capacitor

\subsection{Electrode}

Electrode is the major component of the super-capacitor system and is a site for energy storage. In the electrodeelectrolyte interface the ions will interact with the electrode material and are stored at the active sites of the electrode.

The major parameters of a super-capacitor electrode are porosity, area and conductivity. Such electrode parameters have large influence over the energy density. Optimization of such parameters will lead to high functioning supercapacitor and can lead to the application in existing electronics devices such as laptops and mobile phones.

For the super-capacitor electrode the amount of energy stored depends largely on the electrode surface area as seen from equation $(3,2)$, enormous research is going on globally in search of materials with large surface area for its application in energy storage devices. With increase in the active surface area the number of ions that can be stored by forming Helmholtz double layer will also increase which leads to higher energy density.

Activated carbon is a good candidate for electrode material as it shows adequate capacitance with both aqueous and organic electrolyte. [11], [12]. Major properties of carbon based electrode that makes it suitable for energy storage application are high conductivity, high surface area (1 to $3000 \mathrm{~m} 2$ /gram), good corrosion resistance, high temperature stability, controlled pore structure, compatibility for composite formation and relatively low cost [9].

With the increase in surface area specific capacitance also increases proportionally, however large surface area does not always ensure high specific capacitance, it largely depends on the accessible surface area and pore size of the electrode material. 
Carbons Nanotubes (CNT) bundle possess high porosity because of the network of mesopores so formed by their entanglement, these beautiful network of mesopores improves the penetration of ions increasing the accessible surface area of the electrode [6] [12]. Very high porosity of the electrode has a detrimental effect on the system increasing the electrode resistance furthermore non-uniform pore size results in variable electrode resistance. Size of pores also affects intercalation of ions and determines irreversible capacity involved in the redox reactions. It also determines the formation of decomposition product which gets involved in formation of solid electrolyte interphase (SEI) that is involved in ageing of Super-capacitor [6]. Suggested pore size for optimum performance is 2 to $5 \mathrm{~nm}$ depending upon the size of solvated ions.[13].Porosity is measured by passing nitrogen gas at 77K [6], [1] [12].

Capacitance of super-capacitor also depends on pore radius (b) and distance of approach of ions to carbon substance (d).

$C=\frac{A x}{b \times \operatorname{Ln}\left(\frac{b}{b-A}\right)}$

The other important parameter of super-capacitor electrode is its conductivity and mainly has an effect on Power density. The resistance of electrode material adds up to ESR [14] and limits the power capacity of the super-capacitor system.

In carbon based materials conductivity of Carbon nanotubes is higher as compared to other forms of activated carbon which contributes to its high power density. Conductivity of carbon nanotubes depends on its chiral angle, Arm chair type of carbon nanotubes are metallic in nature whereas zigzag and chiral type are semi conducting, hence arm chair CNTs are preferred for super capacitor system [10], [15], [8].

CNT is widely used as an electrode material because of its characteristic properties which are compatible for energy storage. Wide popularity of CNT based electrode is also because of various available methods to enhance the properties of CNT. Enhancement can be done by functionalization of CNT, introducing defects to CNT and use of CNT with oxides to form composites electrode material.

Functional groups are those groups that are attached to the carbon nanotubes and these groups can take part in redox reaction which contributes to pseudo capacitance. Carbon Nanotubes when treated with nitric acid which is a strong oxidizing agent, the value of specific capacitance is Increased significantly [11] and involves treatment of CNT with strong nitric acid $(6 \mathrm{M})$ at $80^{\circ} \mathrm{C}$ for one hour removing metal catalyst and generating oxygenated functional group [16]. Chemical treatment with $\mathrm{KOH}$ is known to improve the porosity of MWCNT (Multiwall Carbon Nanotubes) [17] and with the introduction of carboxyl group capacitance was increased by 3.2 times which is due to increased hydrophobicity in a MWCNTs based electrode in aqueous electrolyte [16]. Functionalization of CNT with Carboxylic group is also known to improve electrochemical performance in an aqueous electrolyte media like 0.1 molar $\mathrm{H}_{2} \mathrm{SO}_{4}$ [16].

All the defects and roughness of carbon nanotubes are useful in energy storage for EDLC and increasing this in CNT is known to improve the charge storage capacity [18]. Defects can be introduced in CNT by acid treatment $\left(\mathrm{H}_{2} \mathrm{SO}_{4}, \mathrm{HNO}_{3}\right)$ and Ball milling for short period of time which is known to reduce irreversible capacity and improve cycle life due to change in its morphology and orientation [6]. Surface defects in Carbon Nanotubes and the breaking of hemisphere that encloses the nanotube will allow the ions to access the open central canal of carbon nanotubes [11]. The diameter of open central canal should be around 2 to 3 times the diameter of the solvated ion, smaller as well the larger diameter CNT central canal does not contribute much to the ion storage.

CNT and oxide composite will form a hybrid type of electrode that makes use of both EDLC and pseudo capacitance. The open network of mesopores in CNT will allow the ions to easily diffuse into active surface and the oxide composites contribute in pseudo capacitance [10]. The overall capacitance, combination of EDLC and Pseudo capacitance, will be high for such electrode material and will contribute to high power and energy density [11]. Some of such CNT and oxide composites are Ruthenium Oxide and CNTs Composite, $\mathrm{Co}_{3} \mathrm{O}_{4}$ and CNTs composite , $\mathrm{Ni}(\mathrm{OH})_{2}$ and CNT composite, Polymer and CNTs Hybrid Composite, DNA and CNTs Composite [11], [19].

\subsection{Electrolyte}

Electrolyte is a liquid gel or solid that generates ions and allows their movement between the electrodes under the application of electric potential. Electrolyte being the major component of super-capacitor influences its performance, energy density and power density. Depending upon the application and characteristics required for a super-capacitor proper selection of electrolyte is paramount importance for its better performance and efficiency.

Aqueous electrolyte uses water as solvent and is known to be used in high power density application but it is limited by low dissociation potential of water which in turn limits the energy density of system as a whole and most commonly used aqueous electrolytes are $\mathrm{H}_{2} \mathrm{SO}_{4}, \mathrm{H}_{3} \mathrm{PO}_{4}$ (acidic), $\mathrm{KOH}$, $\mathrm{NaOH}$ (Basic), $\mathrm{Li}_{2} \mathrm{SO}_{4}, \mathrm{~K}_{2} \mathrm{SO}_{4}$ and $\mathrm{Na}_{2} \mathrm{SO}_{4}$ (salt). Organic electrolytes makes use of organic compounds like acetonitrile and N-Methyl-2-pyrrolidone (NMP) as a solvent, these electrolytes have higher dissociation potential. Solid electrolytes are the recent advancement in electrolytes and are solids or gels with ions inside them, fabrication process for such electrolytes are easier but the major disadvantage is they have less conductivity in comparison with aqueous electrolyte.

Major parameters that determine the performance of electrolyte are ionic conductivity, ion size, chemical 
reactivity, viscosity [10], solvent molecule, voltage stability window and compatibility with electrode material [20].

Voltage stability window depends on the type of electrolyte and is a major factor affecting energy density of the super capacitor system. For aqueous electrolyte operation voltage is limited to 1 to $1.2 \mathrm{~V}$. Beyond this voltage water gets dissociated into hydrogen and oxygen, $\mathrm{H}_{2}$ is evolved at negative electrode and oxygen at positive electrode. Dissociation of water leads to loss of water (solvent), viscosity of electrolyte increases and ion mobility decreases, which causes super-capacitor's performance to decline [20]. For organic electrolyte voltage window ranges from 2 to $2.4 \mathrm{~V}$ and infers that such system will have greater energy density.

Conductivity of Electrolyte involves ionic conductivity and electrical conductivity. Ionic conductivity governs the movement of ions under the effect of applied electric field and contributes to the power density of the system Equation (4), which increases with increase in ionic mobility. Ionic conductivity depends on concentration of electrolyte; at low concentration of electrolyte number of ions decreases so does the conductivity. If the concentration of electrolyte is high then due to dense ion distribution the collision between solvated ions increase which will affect the ionic mobility and hence the power density. Conductivity of electrolyte depends on ion size and types of solvent and is maximized for optimum concentration. Concentration of electrolyte also relates to diffusion phenomena which contribute to selfdischarge of super-capacitor system. Concentration is also temperature dependent hence the requirement of maintaining a particular temperature while packaging. $\mathrm{H}_{2} \mathrm{SO}_{4}$ has maximum conductivity of $0.8 \mathrm{~s} / \mathrm{cm}$ at $1 \mathrm{M}, 25^{\circ} \mathrm{C}$ and $\mathrm{KOH}$ has maximum conductivity of $0.6 \mathrm{~s} / \mathrm{cm}$ at $6 \mathrm{M}$ and $25{ }^{\circ} \mathrm{C}$ [20].

Size of the hydrated ions should match with the pore size of the carbon based electrode for greater accessibility of surface area and for effective ion adsorption by minimizing the free space [21]. Influence of Ion size was shown by the comparison between $\mathrm{Li}_{2} \mathrm{SO}_{4}, \mathrm{Na}_{2} \mathrm{SO}_{4}$ and $\mathrm{K}_{2} \mathrm{SO}_{4}$ salt [22], it was found that hydrated ionic radius of $\mathrm{K}^{+}$is the smallest [22] and has highest ionic conductivity consequently its accessibility to the inner pores of electrode is much easier than $\mathrm{Na}^{+}$and $\mathrm{Li}^{+}$. As a result, $\mathrm{K}_{2} \mathrm{SO}_{4}$-based electrolyte exhibits the highest capacitance at large scan rates.

Electrolyte should be chemically as well as thermally stable and for the EDLC system electrolyte should not react with other components like electrode, collector and separator, this chemical inertness ensures long cycle life for the supercapacitor system.

However in pseudo capacitance based super-capacitors electrolyte must have chemical compatibility with electrode material for fast reversible redox reaction to occur and should also be compatible with electrode functional groups. In Quinone functionalized carbon nanotube pseudo capacitance is favorable in acidic aqueous electrolyte but the behavior is not the same in basic aqueous electrolyte [20].
Electrolytic compatibility with carbon electrode also has an effect on decomposition of electrolyte. In $\mathrm{LiPF}_{6} /$ $(\mathrm{EC}+\mathrm{EMC}+\mathrm{DMC})$ has decomposition voltage of $4 \mathrm{~V}$ when used for lithium ion battery but it dissociates around 2.7 volt in case of EDLC made of activated carbon [23].

\subsection{Seperator}

Separator is sandwiched layer between the two electrodes to avoid the direct electric contact between them and acts as permeable layer for ion transportation [24].There a need for appropriate selection of separator for the proper functioning of the super capacitor such that adequate energy density, power density, self-discharge and maximum cycle life can be attained. Desirable characteristics like permeability to the ionic flow, appropriate resistance, optimum thickness and porosity, proper interfacial contact, low cost [25], chemical stability and electrolyte retain ability should be exhibited by a good separator.

Thickness of the separator is one of the most determining factor for the better performance .It is found that preferable thickness for separator made of the bacterial cellulose is 1 100 micrometers [24] and for others separator like polyester, polycarbonate and nylon are in the range of 20-350 micrometers [26].There are instances reported in the literature using normal writing paper of thickness 100-3000 micrometers.as a separator. If the thickness is less than 1-10 micrometres than it possess less mechanical strength and easily tear out during fabrication of the super capacitor and if its thickness is more then, the inter-distance between the electrodes increases resulting the low capacitance and also low power density due to increment in the ESR.

Separator must have good pores density to hold the electrolyte and to provide ease flow of the ions through it .Porosity can be measured by gas and liquid absorption method as per ASTM D-2873. and the pores of separator can be analytically studied with the help of Scanning Electronic Microscope [27].Pore size of the separator must be less the size of electrode material to avoid the short circuit and must be greater than the ionic radius of the electrolyte for the proper flow of the ion between the electrode.

\subsection{Collector Plate}

Collector plate provides a connecting link for the electron to flow from active electrode material to the external circuit. Collector plates must exhibit desirable properties like high conductivity for the pathway of electron, adhesive contact with the electrode material, adequate stability during Charging/discharging process and high corrosion resistance behavior for a high performance super capacitor system [28].

Different metallic foil like nickel, aluminum and copper having thickness 20-80 micrometers [28] are being used as collector plates on super capacitors and batteries due to their good conductive properties .Although silver, gold have excellent metallic conductivity they have been rarely used for the collector plate due to economic consideration. 
The major hindrance for the use of metallic foil as collector plate is due to corrosion i.e. when collector plate comes in contact with the electrolyte it gets dissolve electrochemically and leads to corrosion which effects on proper contact with electrode material and finally results on poor performance and less cycle life [29]. Main reason of having corrosion on the electrode is due to low corrosion potential which causes corrosion while charging at high voltage, reactivity of the electrolyte towards collector plate, presence of impurities, formation of gases like oxygen which enhance the corrosion process. In order to avoid the corrosion, there should be essential of conductive layer insertion between the collector plate and electrode material. Conductive layer can either be coated on the collector plate like zirconium nitride, titanium nitride or distinct polymer layer insertion between collector-electrode gaps. It is found that preferable resistance for conductive layer is less than $100 \mathrm{ohm}$ per $\mathrm{cm}$. Conductive polymer like polypropylene sheet containing $50 \%$ fine carbon particle by volume showed the better stable and conductivity as well as wear resistance [30]

Improper contact leads to the increase the interface resistance which degrades the power density; this is mainly avoided by acid treating the collector plate like $\mathrm{H}_{2} \mathrm{SO}_{4}$ \& $\mathrm{HNO}_{3}$ to remove the impurities oxide and irregularities [28].

\section{PERFORMANCE CHARECTERIZTION}

Characterization is a vital step for optimization and validation of super-capacitor system, all components of super-capacitor system individually contributes toward its performance however the synergic effect for the system should be accounted for its overall characterization. Many characterization techniques such as cyclic voltammetry, Electrochemical Impedance Spectroscopy (ESI) and Charge Discharge Curve are vital for performance evaluation of super-capacitor [31].

To evaluate the performance of super-capacitor system three major parameters considered are cell capacitance, operating voltage and equivalent series resistance (ESR) but some additional parameters like mass loading, electrode thickness, electrode density, packaging, current collector and dwelling time should also be taken into account [31].

\subsection{Capacitance}

Capacitance is a measure of amount of electric charge that is stored under the applied potential and is a ratio of amount of charge stored $(\Delta \mathrm{Q})$ to the applied potential $(\Delta \mathrm{V})$.

$C=\frac{a Q}{\Delta V}$

Specific capacitance which is capacitance per unit mass or area or volume and is generally preferred while specifying a super-capacitor, it is a ratio of amount of charge stored per unit applied voltage per unit mass or area or volume.

$C s=\frac{\Delta Q}{\Delta W \times \sigma}$
Where, $\sigma$ is mass or area or volume.

Specific capacitance for carbon based electrode material is generally $100 \mathrm{~F} / \mathrm{g}$ and $70 \mathrm{~F} / \mathrm{cm}^{2}$ with organic electrolyte [31].

Capacitance in a generalized way is expressed as

$C=\frac{\Delta Q}{\Delta V}=\int \frac{I \mathrm{xat}}{2 V}$

If the current maintained constant during charging and discharging process then above equation can be expressed as

$C=\frac{1 \times \Delta 4}{2 W}$

Experimentally evaluation of capacitance is done by calculating area under charge discharge curve in cyclic voltammetry. Specific capacitance can be calculated from equation (8). For a given capacitance 'c' specific capacitance depends upon a for which there is no standard process of evaluation [32].

Major factor affecting the capacitance measurement of a super-capacitor are type of experimental setups whether double electrode or triple electrode setup, electrode configuration namely single electrode, symmetric electrode or asymmetric electrode [31]. Specific capacitance also depends on mass loading and electrode thickness [31]. It is found that mass loading for smooth functioning of supercapacitor should be at least $55 \mathrm{mg} / \mathrm{cm}^{2}$ and thickness should be around 50 to $200 \mu \mathrm{m}$ [31].

\subsection{Equivalent Series Resistance (ESR)}

In a super-capacitor system ESR is the total resistance of the system contributed by its different components namely electrode resistance, electrolyte resistance, separator resistance and contact resistance at the interface of collector and electrode. ESR has an effect on power performance and energy efficiency of the system.

Generally an EDLC super-capacitor system is physically modeled with two capacitors in series connected through a resistor and resistance of this resistor is numerically equal to ESR.

ESR can be evaluated by IR (ohmic voltage) drop method or steady voltage variation at initial stage while discharging [31],

$\operatorname{Resr}=\frac{\Delta V}{\Delta \mathscr{V}}$

Where $\mathrm{v}$ is voltage and I is current for IR drop. A large current in charging and discharging reduces the value of ESR.

The major factor influencing the ESR is dwelling time and size of super-capacitor. For large super-capacitor steady stage voltage variation method is used whereas for small 
size of super-capacitor IR drop method is used [31]. The effect of dwelling time is not fully understood and has further scope for research.

\subsection{Operating Voltage}

Operating voltage $\left(\mathrm{V}_{\mathrm{o}}\right)$ is another determining parameter for performance of super-capacitor system and is the range of voltage in which it can perform without degradation. Operating voltage can be evaluated using both cyclic voltammetry and charge-discharge curve [31] [28] [33]. In the process of evaluating the operating voltage, the system may get spoiled hence an expedient method is advised, where electrode potential is slowly increased till spike is seen in $\mathrm{C}-\mathrm{V}$ or charge-discharge curve and the corresponding voltage represents the maximum operating voltage. Room temperature ionic liquid (RTLI) is best suited electrolyte from operating voltage point of view with a range of 3.0 to 6.0 volts [31].

\subsection{Time Constant}

Time constant for super-capacitor system is the product of ESR and specific capacitance $\left(\mathrm{C}_{\mathrm{s}}\right)$ and determines the responsiveness of the system [28].

$T=R_{e s r} \times C_{s}$

Smaller the value of time constant more is the responsiveness of such super-capacitor system, for a commercial super-capacitor system $\mathrm{T}$ ranges from 0.5 to 3.6 seconds [31]. $\mathrm{T}$ is depends on components of supercapacitor, its material and manufacturing process. Commercial super-capacitor like Maxwell, Ness cap and JSR micro have time constants respectively $0.55 \mathrm{~s}, 1.1 \mathrm{~s}$ and $3.5 \mathrm{~s}$ [31].

\subsection{Power Density and Energy Density}

The parameters that are related to end application of all kind of energy storage devices are power density and energy density and are extensively researched to improve the performance of the systems. The power density of capacitor is comparatively high whereas the energy density is higher for battery; super-capacitors have these parameters in between battery and capacitors. Hence enormous research is going on in developing super-capacitors which can be used in tandem with battery and capacitors. [3]

\subsubsection{Power Density}

Power density for a super capacitor system is defined as amount of energy that can be delivered by the system per unit time and given by the equation below [34].

$\mathrm{P}=\frac{v^{2}}{4 \sigma \mathrm{R}_{\mathrm{Gar}}}$

From equation it is evident that power density is maximized only when external load is equal to the ESR resistance a condition for matched loading. Most of the time external load resistance will be different from ESR, in such cases the above method is inappropriate and the methods used are DOE freedom car [36] and pulse energy efficiency [35] criteria. The maximum theoretical power density calculated by above equation is used for comparing various energy storage systems but the actual power that can be harnessed is different from maximum power rating [31].

\subsubsection{ENERGY DENSITY}

Energy density is the amount of energy that can be stored by super-capacitor per unit mass or volume. Electrically stored charge during charging or discharging can be evaluated by integrating the $\mathrm{C}-\mathrm{V}$ curve that is generated from cyclic voltammetry Stored energy is evaluated from charging curve and deliverable energy is evaluated from discharging curve [31] [34].

Energy efficiency for the system is used for comparing various systems and is the ratio of deliverable energy to stored energy [31].

For a EDLC system

$\mathrm{E}=\frac{1}{2} \times \mathrm{V} \times \mathrm{q}$

This can be modified as

$\mathrm{E}=\frac{\mathrm{cx}^{2}}{2 \times \sigma}$

Energy density and power density is related with each other through time constant,

$\frac{\mathrm{E}}{\mathrm{F}}=2 \times \mathrm{T} \ldots \ldots(16)[31]$

Energy density can be improved either by increasing voltage or capacitance, if capacitance is increased then time constant ' $\mathrm{T}$ ' will also increase which will cause decrease in responsiveness of the system with the assumption of constant ESR. If the voltage is increased then both energy density and power density is increased with time constant unchanged, this proves solvent selection in electrolyte is important because the type of solvent selected limits the operating voltage. Energy density should be improved as much as possible by using new electrode material or by optimizing existing systems however time constant and power density should also be taken care since these parameters are interrelated and complex [31] [34].

\subsection{Self Discharge}

Leakage popularly known as self-discharge is an important parameter to estimate the capacity of system to maintain the rated potential at idle condition [36]. Self-discharge is measured in terms of compensating current that has to be applied to hold charge in fully charged capacitor after 72 hours [31]. This leakage occurs due to reasons like diffusion of ions from higher concentration to lower concentration, leakage of charge across double layer at the electrodeelectrolyte interphase, temperature of the system and initial 
voltage [37]. Diffusion process dominates the self-discharge for 8 hours form the beginning of discharge process [31].

When potential is applied on the electrode it stimulates the ion movement and at the end of the process the positive and negative charges gets separated, in between some faradic reaction occurs and causes local increase in concentration contributing to the diffusion process. This local build-up of ionic concentration occurs only above threshold voltage and current flowing through the system. Leakage of charge from electrochemical double layer is mainly due to the impurities present which causes the gradual loss of cell potential [28].

Self-discharge which contributes to the charge loss is still an unsolved problem in this field and it seems inevitable and hence commercial super-capacitors are not sold in fully charged condition.

\section{CONCLUSION}

High performance super capacitor depends largely on material selected for its components mainly electrode, electrolyte, separator and collector plate. Energy density, power density and specific capacitance are interrelated and mainly depend on electrode material .Active carbon including CNT and graphene are good choices for positive and negative electrode. Aqueous electrolyte works well with these electrode materials however operating voltage is limited. Solid electrolyte is the better option but their performance in terms of power density and cycle life is a matter of concern.

\section{REFERENCES}

[1]: Xin lee and Bingqing Wei (2013). Super Capacitor Based On Nanostructured Carbon. ELSEVIER: Nano Energy (2013)2,159-173.

[2]: Dr. Martin Winter and Dr. Rolph J. Brodd (2004). What are Batteries, Fuel Cells and Super Capacitors?. Chem. Rev 2004, Vol 104, No. 10.

[3]: B. E. Conway. (1991). Transition from "Supercapacitor" to "Battery" Behavior in Electrochemical Energy Storage. Journal Of Electrochemical Society, DOI: 10.1149/1.2085829.

[4]: T.R. Ceompton. (2000). Battery Refernce Book. Reed Educational and Professional Publishing Ltd 1990, 1995, 2000. ISBN $075064625 \mathrm{X}$

[5]: Christopher D. Rahn and Chao-Yang Wang (2013). Battery Systems Engineering. A John Wiley \& Sons, Ltd., Publication.

[6]: Elzbieta Frackowiak and Francois Beguin. Electrochemical storage of energy in carbon nanotubes and nanostructured carbon. Carbon: Volume 40, Issue 10, August 2002, Pages 1775-1787, DOI: 10.1016/S00086223(02)00045-3

[7]: B.E. Conway. (1999). Similarities and differences between Supercapacitors and Batteries for Storing Electrical energy. Electrochemical Supercapacitors: Scientific Fundamentals and Technological Applications, Chapter2. KLUWER ACADEMICS/ PLENUM PUBLISHERS.
[8]: M.S. Dresshelhaus, G. Dresshelhaus and Ph. Avouris(eds.). Introduction to Carbon Materials Research. Topics in Applied Physics, Volume 80, 1-9, ISSN electronic edition: 1437-o859

[9]: ]: A.G. Pandolfo and A.F. Hollenkamp (2006). Carbon Properties and their role in Supercapacitors. ELSEVIER: Journal of Power Sources 157 (2006), 11-27

[10]: Francois Béguin, Volker Presser, Andrea Balducci and Elzbieta Frackowiak (2014). Carbons and Electrolytes for Advanced Supercapacitors. Advanced Materials, DOI: 10.1002/adma.201304137.

[11]: Hui Pan, Jianyi Li, Yuan Ping Feng. 2010. Carbon Nanotubes for Supercapacitor. Nanoscale Res Lett (2010) 5:654-668, DOI 10.1007/s11671-009-9508-2

[12]: Chmiola, John and Yushin , G. and Gogotsi, Y. and Portet, Christele and Simon, Patrice and Taberna, PierreLouis . (2006). Anomalous Increase in Carbon Capacitance at Pore Sizes Less Than 1 Nanometer. Science Magazine, vol. 313 ( $n^{\circ}$ 5794). pp. 1760-1763. ISSN 0036-8075

[13]: Patrice Simon \& Yury Gogotsi. (2008). Materials for electrochemical capacitors. Nature: Nature Materials 7, 845 - 854 (2008) doi:10.1038/nmat2297

[14]: B.E. Conway (1999). Elements of electrostatics involved in treatment of double layer and ions at Capacitor Electrode Interphases. Electrochemical Supercapacitors: Scientific Fundamentals and Technological Applications, Chapter 4 . KLUWER ACADEMICS/ PLENUM PUBLISHERS.

[15]: Meisam Valizadeh Kiamahalleh, Sharif Hussein Sharif Zein, Ghasem Najafpour, Suhairi Abd Sata and Surani Buniran (2012). Multiwalled carbon nanotubes based nanocomposites for supercapacitors: A review of electrode materials. NANO: Brief Reports and Reviews Vol. 7, No. 2 (2012) 1230002 (27 pages) CWorld Scientific Publishing Company, DOI: 10.1142/S1793292012300022.

[16]: E. Frackowiak, K. Metenier, V. Bertagna and F. Beguin. Supercapacitor electrodes from multiwalled carbon nanotubes. Appl. Phys. Lett. 77, 2421 (2000), DOI: 10.1063/1.1290146

[17]: E. Frackowiak, S. Delpeux, K. Jurewicz, K. Szostak, D. CazorlaAmoros and F. Beguin. Chem. Phys. Lett. 261, 35 (2002)

[18]: M.S. Dresshelhaus, G. Dresshelhaus and Ph. Avouris(eds.). Electronic Properties, Junctions, and Defects of Carbon Nanotubes. Topics in Applied Physics, Volume 80, 113-145, ISSN electronic edition: 1437-o859

[19]: Meisam Valizadeh Kiamahalleh, Sharif Hussein Sharif Zein, Ghasem Najafpour, Suhairi Abd Sata and Surani Buniran (2012). Multiwalled carbon nanotubes based nanocomposites for supercapacitors: A review of electrode materials. NANO: Brief Reports and Reviews Vol. 7, No. 2 (2012) 1230002 (27 pages) CWorld Scientific Publishing Company, DOI: 10.1142/S1793292012300022.

[20]: Cheng Zhong, Yida Deng, Wenbin Hu, Jinli Qiaoc, Lei Zhang and Jiujun Zhang (2015). A review of electrolyte materials and compositions for electrochemical supercapacitors. Royal Society of Chemistry, DOI: $10.1039 / \mathrm{c} 5 \mathrm{cs} 00303 \mathrm{~b}$

[21]: Celine Largeot, Cristelle Portet, John Chmiola, Pierre Louis Taberna, Yury Gogotsi and Patrice Simo (2008). 
Relation between the Ion Size and Pore Size for an Electric Double-Layer Capacitor. Journal of the American Chemical Society. 2008, DOI:10.1021/ja7106178

[22]: Q.T. Qu, B. Wang, L.C. Yang, Y. Shi, S. Tian and Y.P. Wu. 2008. Study on electrochemical performance of activated carbon in aqueous Li2SO4, Na2SO4 and $\mathrm{K} 2 \mathrm{SO} 4$ electrolytes. Electrochemistry Communications 10 (2008) 1652-1655, doi:10.1016/j.elecom.2008.08.020

[23]: ZHOU Shao-yun, LI Xin-hai, WANG Zhi-Xing, GUO Hua-jun and PENG Wen-jie. 2007. Effect of activated carbon and electrolyte on properties of supercapacitor. Transactions of Nonferrous Metals Society of China Vol I7 (2OO7) 1328- 1333.

[24]: Electric double-layer capacitor and method of making a separator therefor US $6190501 \mathrm{~B} 1$

[25]: Aquagel electrode separator for use in batteries and supercapacitors US 5402306 A

[26]: Boris Dyatkin, Volker Presser, Min Heon, Maria R. Lukatskaya, Majid Beidaghi, and Yury Gogotsi. Development of a Green Supercapacitor Composed Entirely of Environmentally Friendly Materials. DOI: $10.1002 /$ cssc. 201300852

[27]: Separator (electricity), available in Wikipedia. https://en.m.wikipedia.org/wiki/Separator_(electricity)

[28]: Aiping Yu, Victor Chabot, Jiujun Zhang. ELECTROCHEMICAL SUPERCAPACITORS FOR ENERGY STORAGE AND DELIVERY.CRC Press Taylor and Francis group, 6000 Broken Sound Parkway NW, Suite 300 .

[29]: ]: Samvel Avakovich Kazaryan, Valery Pavlovich Nedoshivin, Vladimir Alexandrovich Kazarov, Gamir Galievich Kharisov, Sergey Vitalievich Litvinenko and Sergey Nikolaevich Razumov (2007).Current collector for a double electric layer capacitor. [30]: Shankar Dasgupta, James K. Jacobs (1995. Current collector for lithium ion battery. Patent Number: US5464706 A

[31]: Sanliang Zhang and Ning Pan (2014). Supercapacitors Performance Evaluation. Adv. Energy Mater. 2014, 1401401. DOI: 10.1002/aenm.201401401

[32]: B.E. Conway (1999). Behavior of Dielectrics in Capacitors and theories of Dielectric Polarization. Electrochemical Supercapacitors: Scientific Fundamentals and Technological Applications, Chapter 5. KLUWER ACADEMICS/ PLENUM PUBLISHERS.

[33 B.E Conway (1964). Theory and Principles of electrode processes. Ronald Press, New York.

[34]: B.E. Conway (1999). Energy Density and Power Density of Electrical Energy Storage Devices. Electrochemical Supercapacitors: Scientific Fundamentals and Technological Applications, Chapter 15. KLUWER ACADEMICS/ PLENUM PUBLISHERS.

35]: A. Burke and M. Miller (2011). J. Power Sources 2011, 196, 514.

[36]: B.E. Conway (1999). Self-Discharge of Electrochemical Capacitors in Relation to that of Batteries. Electrochemical Supercapacitors: Scientific Fundamentals and Technological Applications, Chapter 6. KLUWER ACADEMICS/ PLENUM PUBLISHERS.
[37]: B.E. Conway (1999).The Double Layer Of Capacitor Electrode Interface: Its structure and Capacitance. Electrochemical Supercapacitors: Scientific Fundamentals and Technological Applications, Chapter 6. KLUWER ACADEMICS/ PLENUM PUBLISHERS.

\section{ACKNOWLEDGMENT}

We wish to express sincere gratitude to the management, Principal and faculty of M.S. Ramaiah Institute of Technology for their support and encouragement.

Special thanks to Rajesh, Srinivas, Baby and Babu, technical staff of mechanical department, MSRIT for their support.

Our sincere appreciation to Mr. Karthik and Mr. Ashutosh, Ex-student of MSRIT for their unquestioned support towards this work. 